\title{
Estudio Histoquímico de la Enzima NADH-TR en Músculo Frontal de Conejos Norfolk (Oryctolagus cuniculus)
}

\author{
Histochemical Study of Enzyme in Frontal Muscle of \\ Norfolk Rabbit (Oryctolagus cuniculus)
}

"André Hebling; "Marcelo Augusto M. Esquisatto \& **Heloisa A. de Lima Castro

HEbling, A.; ESQUISATTO. M. A. M. \& CASTRO, L. H. Estudio histoqímico de la enzima NADH-TR en músculo frontal de conejos Norfolk (Oryctolagus cuniculus). Int. J. Morphol., 24(2):151-154, 2006.

RESUMEN: El músculo frontal humano posee dos vientres formando junto, al músculo occipital y la gálea aponeurótica, el músculo occipitofrontal. Como músculo estriado esquelético, el músculo frontal puede presentar fibras con alta intensidad de oxidación (tipo I) y con baja intensidad de oxidación (tipo II). El objetivo de este trabajo fue la determinación, a través de la reacción histoquímica para nicotinamide adenine dinucleotide tetra-zolium redutase (NADH-TR), la distribución de fibras de tipos I y II del músculo frontal de conejos de la raza Norfolk inglesa, del sexo femenino, con edad de seis a ocho meses, pesando de 2,8 a 3,1 Kg. De un total de 1010 fibras estudiadas, la proporción encontrada fue de 33,8\% para el tipo I, de 17,4\% para el tipo IIA, y de 48,8\% para el tipo IIB. La mayoría de las fibras encontradas era del tipo II, revelando la intensa actividad de este músculo en la mímica facial. Las fibras de tipo II presentan baja intensidad oxidativa, pudiendo así sufrir fatiga muscular. Estas conclusiones pueden ser un aporte en estudios para entender los procesos patológicos que pueden ocurrir en este músculo.

PALABRAS CLAVE: Histoquímica; Músculo frontal; Tipos de fibras.

\section{INTRODUCCIÓN}

El músculo frontal actúa en la mímica facial formando, junto con el músculo occipital y la unión con la galea aponeurótica, el músculo occipitofrontal (Figun, 1986; DiDio, 1999). De acuerdo con Berzin (1973), los vientres occipital y frontal del músculo occipitofrontal tienen acción independiente, a pesar de la galea aponeurótica. El vientre frontal, al mismo tiempo en que levanta la ceja homolateral, tracciona la galea aponeurótica hacia adelante y, por tanto, es responsable la formación de arrugas transversales en la región frontal. El vientre occipital es responsable de traccionar la galea aponeurótica para atrás, al mismo tiempo que participa de la sonrisa y puede o no participar en movimientos del pabellón de la oreja.

La histoquímica, o más precisamente, la histoenzimología de los músculos esqueléticos ha sido utilizada con éxito en la diferenciación de grupos de fibras funcionalmente distintas. Esto se debe a la gran concentración de enzimas oxirreductasas en los tejidos musculares, principalmente las de tipo diaforasa, como la nicotinamida adenina dinucleótida tetra-zolium redutasa (NADH-TR) (Dubowitz \& Brooke, 1973).

La aplicación del método de la NADH-TR al estudio del tejido muscular esquelético, permitió la diferenciación de varios tipos de fibras metabólicamente distintas. En la práctica, el análisis de la morfología, histoquímica, morfometría y la distribución de las fibras, han proporcionado una valiosa colaboración para el diagnóstico de numerosas miopatías. Con esta reacción, las fibras musculares pueden ser clasificadas en tipo I, reacción oxidativa fuerte, y tipo II, reacción oxidativa débil (Dubowitz et al., 1985).

Debido a la ocurrencia frecuente de procesos patológicos que inciden en el M. frontal y a la ausencia de estudios histoquímicas sobre el mismo en la literatura, este trabajo tiene como objetivo la diferenciación histoquímica de los grupos de fibras de ese músculo en conejos, así como cuantificar la distribución media de sus fibras en una población de animales criados en cautiverio.

\footnotetext{
* Disciplina de Anatomia Humana, Centro Universitário Hermínio Ometto- UNIARARAS, Araras, São Paulo, Brasil.

${ }^{* *}$ Departamento de Morfologia, Faculdade de Odontologia, Universidade Estadual de Campinas - UNICAMP, Piracicaba, São Paulo, Brasil.
} 


\section{MATERIAL Y MÉTODO}

Fueron estudiados 10 conejos adultos, hembras, de la raza Norfolk inglesa, con 6 a 8 meses de edad, con peso entre 2.8 a $3.1 \mathrm{Kg}$, mantenidos en cautiverio, alimentados con ración adecuada y agua ad libitum.

Las disecciones, biopsias e histoquímica de los músculos frontales de los animales seleccionados fueron efectuadas en el Laboratorio Experimental del Departamiento de Neurología de la Facultad de Medicina de Botucatu, de la Universidad Estadual de São Paulo - UNESP.

La recolección del material y el manejo de los animales fueron realizados de acuerdo con los principios éticos preconizados por el Colegio Brasileño de Experimentación.

Los animales fueron anestesiados con Hypnol al 3\% en la dosis de $30 \mathrm{mg} / \mathrm{Kg}$ de peso para la extracción de las muestras y, en seguida, sacrificados por inhalación con éter. Un fragmento de $15 \mathrm{~mm}^{2}$ fue retirado del vientre izquierdo del músculo frontal y depositado en una placa de Petri, identificado y remitido inmediatamente para su procesamiento.
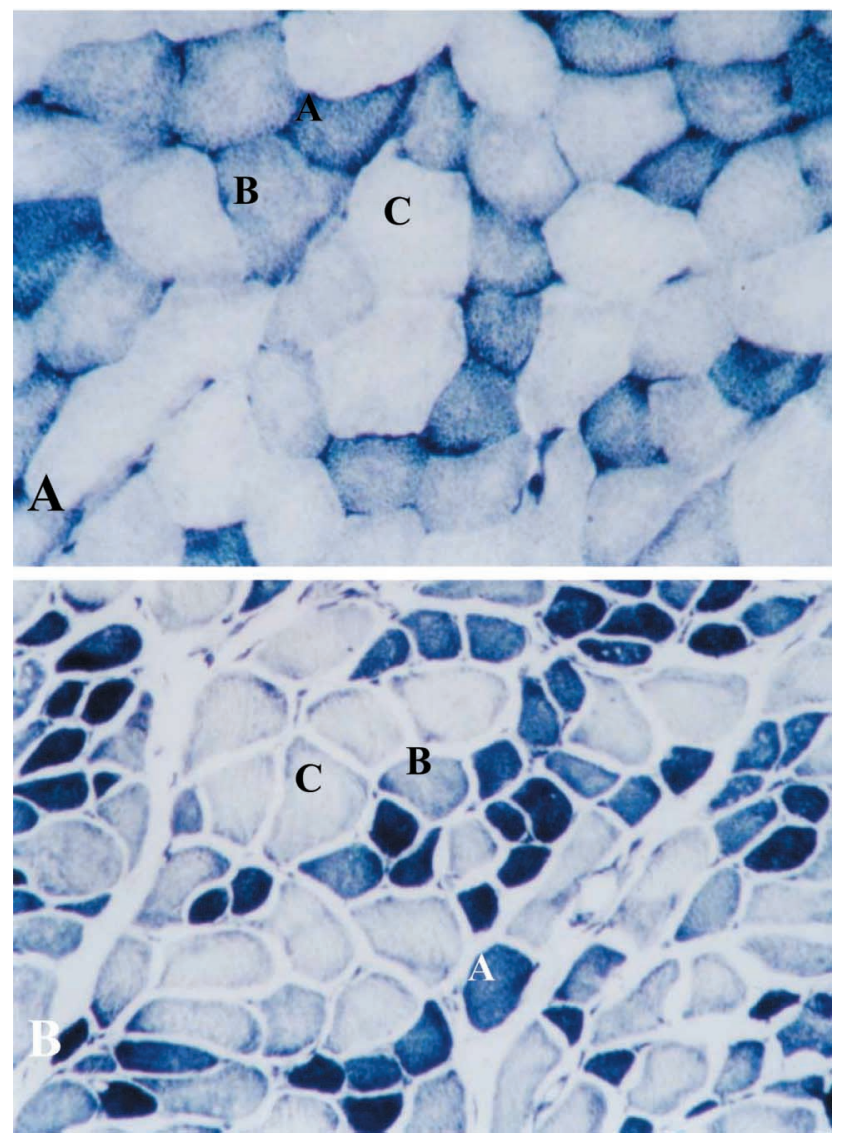

Los muestras de músculo fueron congeladas en $n$ hexana, enfriadas a $-70{ }^{\circ} \mathrm{C}$ con ayuda de nitrógeno líquido. Luego, fueron transferidas a una cámara de criostato Leica modelo CM 1800, a $-20^{\circ} \mathrm{C}$, donde permanecieron durante 40 minutos para llegar al equilibrio térmico. Fueron fijadas en soporte metálico (Tissue-Tek-OCT), y sometidas a microtomía para la obtención de cortes con $8 \mu \mathrm{m}$ de espesor. Los cortes fueron llevados a la estufa a $37^{\circ} \mathrm{C}$, donde permanecieron por 10 minutos. Posteriormente, fueron sometidos a la reacción histoquímica para la identificación de la enzima nicotinamida-dinucleótidea tetrazolium reductasa (NADH-TR), de acuerdo con los métodos descritos por Dubowitz et al.

Los cortes fueron fotografiados en fotomicroscopio marca Zeiss, modelo Mc 80 DX, con aumento óptico de 10 $X$. Las imágenes de la reacción para NADH-TR fueron ampliadas 180 veces y divididas en 4 cuadrantes iguales, para facilitar el conteo y la identificación de las fibras. El total de fibras tipo I, IIA y IIB de cada cuadrante fue determinado. La suma de los totales de tipos fibrilares de cada cuadrante determinó el número total de fibras de la muestra.

\section{RESULTADOS}

La Fig. 1 ilustra las muestras marcadas por NADHTR, mostrando la distribución de los diversos tipos de fibras musculares. La distribución de los tipos de fibras en los muestras analizadas está resumida en la Tabla I.

Tabla I. Distribuciones absoluta y relativa de los tipos de fibras encontrados en el M. frontal de conejos de la raza Norfolk (Oryctolagus cuniculus).

\begin{tabular}{ccc}
\hline Tipos de Fibras & Número & Porcentaje \\
I & 341 & 33,8 \\
II A & 176 & 17,4 \\
II B & 493 & 48,8 \\
TOTAL & 1010 & 100,0 \\
\hline
\end{tabular}

Fig. 1. Microfotografias de espécimenes 1 (A) y 8 (B) después de la reacción para NADH-TR. Observe las diferencias en la distribución de los tipos de fibras entre los animales estudiados. A - tipo I; $\mathrm{B}$ - tipo IIA y C - tipo IIB. $180 \mathrm{X}$. 


\section{DISCUSIÓN}

Debido a su posición anatómica y a su actividad en la mímica facial, el músculo frontal está entre los músculos más exigidos en el estudio del movimiento del rostro. Por tanto, se torna relevante el análisis de los tipos de fibras que forman esos músculos, y consecuentemente, relacionar este análisis con las posibilidades funcionales de los mismos.

Fueron escogidos conejos debido al manejo fácil y a las características anatómicas y funcionales semejantes a las de los seres humanos.

Los estudios histoquímicos de los músculos de la mímica son poco mencionados en la literatura. En especial, son inexistentes los que se refieren al músculo frontal. Sin embargo, son encontrados algunos relatos relacionados a actividad electromiográfica (Bérzin).

De un total de 1010 fibras musculares marcadas en los ocho especímenes estudiados, la mayor parte $(66,2 \%)$ fue del tipo II y $33,8 \%$ fue del tipo I. En las fibras de Tipo II es posible diferenciar fibras de tipos A $(17,4 \%)$ y B $(48,8 \%)$, lo que se muestra en la Tabla I).

Estos datos indican un músculo con intensa actividad fisiológica. Esto es corroborado con los registros electromiográficos durante la mímica facial (Bérzin).
Las fibras de tipo II presentan baja intensidad oxidativa, siendo por tanto susceptibles a presentar fatiga muscular. Este resultado puede ser útil en estudios fisiopatológicos de las miopatías de este músculo.

La diferenciación de los tres tipos de fibras musculares a través de la reacción oxidativa de NADH-TR es la más frecuentemente utilizada, aunque no responda a todos los problemas funcionales envueltos en la fisiología del músculo (Dubowitz \& Brooke; Dubowitz et al.). Además, varios relatos en la literatura indican que la tipificación y cuantificación de los tipos no abarcan la gran variabilidad existente entre músculos de un mismo animal y de animales diferentes (Ogata, 1958 a, b y c; Stein \& Padykula, 1962; Nishiyama, 1966; Guth \& Samaha, 1969; Peter et al., 1972).

Para minimizar este problema, se hace necesario asociar otras medidas morfométricas y reacciones histoquímicas para obtener una mejor análisis global del músculo.

Finalmente, nuestro trabajo es una aporte para la literatura, contribuyendo con la descripción cualitativa y cuantitativa de los tipos de fibra encontrados en el M. frontal de conejos de la raza Norfolk inglesa, y la confirmación morfológica de su actividad predominantemente de baja intensidad oxidativa.

AGRADECIMIENTOS: CNPq/MCT-Brasil y PROPESq/ UNIARARAS, Brasil.

HEbling, A.; ESQUiSATTO. M. A. M. \& CASTRO, L. H. Histochemical study of enzyme in frontal muscle of Norfolk rabbit (Oryctolagus cuniculus). Int. J. Morphol., 24(2):151-154, 2006.

SUMMARY: The human frontal muscles has two bellies, forming, together with the occipital muscle and the aponeurotic galea, the occipitofrontal muscle. Being a striated skeletal muscle, the frontal muscle can show muscular fibers with high intensity oxidation (type I) and with low intensity oxidation (type II). A histochemical process using organic and inorganic chemical reactions allows us to obtain a product that is visible through optical microscopy. The purpose of this paper is to determine, by means of the histochemical process of the Nicotinamide Adenine Dinucleotide Tetra-zolium Reductase (NADH-TR) reaction the distribution of the number of the type I and II frontal muscle fibers of female, six to eight months old, 2.8 to $3.1 \mathrm{Kg}$ English Norfolk rabbits. In 1010 fibers studied, we found $33.8 \%$ type I, $17.4 \%$ type IIA and $48.8 \%$ type IIB. The majority of fibers were type II, demonstrating this muscle's intense activity during facial mimicking. Type II fibers showed a reduced oxidative intensity, which made them subject to muscular fatigue. These findings may be of help in the study to understanding the pathological process that may occur in this muscle.

KEY WORDS: Histochemistry, 2. Frontal muscle; Fiber types.

\section{REFERENCIAS BIBLIOGRÁFICAS}

Bérzin, F. M. Occipitofrontalis: Análise eletromiográfica e seu significado funcional. Tese (Doutorado em Anatomia) - Faculdade de Odontologia de Piracicaba, Universidade Estadual de Campinas, 1973.
DiDio, L. J. A. Tratado de Anatomia Aplicada. São Paulo, Póluss Editorial, 1999.

Dubowitz, V. \& Brooke, M. H. Muscle biopsy: a modern 
approach. London-UK, Saunders, 1973.

Dubowitz, V.; Sewry, C. A. \& Fitzsimons, R. B. Muscle Biopsy: A Practical Approach. $2^{\text {th }}$ ed. London-UK, Baillière Tindall, 1985.

Figun, M. E. Anatomia Bucal. $3^{\circ}$ ed São Paulo, Médica Panamericana, 1986.

Guth, L. \& Samaha, F. J. Qualitative differences between actomyosin ATPase of slow and fast mammalian muscle. Exp. Neurology, 25 (2):138-52, 1969.

Nishiyama, A. Histochemical studies on the red and white and intermediate muscle fibers of some skeletal muscles. III- Histochemical demonstration of oxidate enzymes, phosphorylase and glycogen in respiratory muscle fibers. Acta Medica Okayama, 20(1):1378-86, 1966.

Ogata, T. A Histochemical study of the red and white muscle fibers.Part I: Activity of the succinoxidase system in muscle fibers. Acta Medica Okayama, 12(1):216-27, 1958a.

Ogata, T. A Histochemical study of red and white muscle fibers. Part II: Activity of the cytochrome oxidase in muscle fibers. Acta Medica Okayama 12(1):228-32, $1958 b$.

Ogata, T. A Histochemical study of the red and white muscle fibers.Part III: Activity of the diphosphopyridine nucleotide diaphorase and triphosphopyridine nucleotide diaphorase in muscle fibers. Acta Medica Okayama 12(1):233-40,1958c.

Peter, J. B.;Barnard, R. J.; Edgerton, V. R.; Gilespie, C. A. \& Stempel, K. E. Metabolic Profiles of Three Fiber Types of Skeletal Muscle in guinea pigs and rabbits. Biochemistry, 11(1):2627-33, 1972.

Stein, J. M. \& Padykula, H. Histochemical classification of individual skeletal muscle fibers of the rat. Am. J. Anat., 110 (1):103-24, 1962.
Dirección para correspondencia:

Prof. André Hebling

Disciplina de Anatomia Humana

Centro Universitário Herminio Ometto

UNIARARAS

$\mathcal{A v}$. Dr. Maximiliano Baruto, 500 gd. Universitário

CEP 13607-339

Araras $/ \mathcal{S P}$

BRASIL

Tel/Fax : 055019 3543-1517

Email: andrehe6ling@uniararas.6r

Recibido : 26-12-2005

Aceptado: 02-03-2006 